\title{
PAROXYSMAL TACHYCARDIA
}

\author{
WITH REFERENCE TO NOMOTOPIC TACHYCARDIA AND THE \\ RÔLE OF THE EXTRINSIC CARDIAC NERVES*
}

\author{
ALFRED M. WEDD, M.D. \\ PITTSBURGH
}

Of the recognized disorders of the cardiac mechanism, none possess greater interest than the paroxysmal tachycardias. This affection has been recognized as a clinical entity for more than thirty years, and the condition has been produced experimentally by various means. Two questions have recently been revived; one, concerning sites of origin, especially the possibility of a paroxysm arising at the sinoatrial node (nomotopic tachycardia) and the other, concerning the rôle of the extrinsic nerves of the heart. Herewith $I$ wish to report six cases ${ }^{1}$ of paroxysmal tachycardia which illustrate the moot points and the variety of clinical conditions in which the disorder occurs.

\section{REPORT OF CASES}

CASe 1.--J. W., aged 19 years, student. This patient was first seen Dec. 30, 1919, when he came for examination because of unduly rapid and forceful heart action which frequently occurred following exercise. He says they have been present as long as he can remember, and his mother recalls attacks as early as 5 years when the child often ran to her, frightened because of the abnormal cardiac activity. An aunt has told him that when he was a small boy she had noticed that his heart was extremely rapid when at play. Rarely, pounding of the heart is experienced at night but usually there is no consciousness of the heart except during the attacks. The paroxysms are often related to exercise but from consideration of many attacks it is clear that they are not dependent on the total energy expended but rather follow sudden or spasmodic effort. Attacks have occurred irregularly during gymnasium exercises, following certain maneuvers in military drill, swimming, especially just after plunging into cold water, after a sudden spurt for a car or coughing. But in carefully planned gymnasium work and hard labor in the hay field during the summer the pulse rate does not rise above 80 . At the laboratory attacks have followed running up and down stairs but at other times this exercise or hopping until dyspnea necessitated rest did not bring on a paroxysm. The emotional factor is seen in the occurrence of paroxysms while dancing and while watching an exciting football match. These attacks always begin suddenly and cease abruptly; according to the patient, "it is just like shifting gears on a car." The duration is from a few minutes to two hours. He thinks he often stops an attack by holding his breath or throwing himself suddenly across a bed or convenient object, and says that recently an attack ceased promptly after pressure on the right eyeball. During the attack the heart beats rapidly and

* From the Electrocardiographic Laboratory of Mercy Hospital.

1. These patients were referred by Drs. J. A. Lichty (Cases 1. 4 and 5). E. M. Frost (Case 2), J. I. Johnston (Case 3), and G. L. Hays (Case 6), to whom I express my indebtedness. 


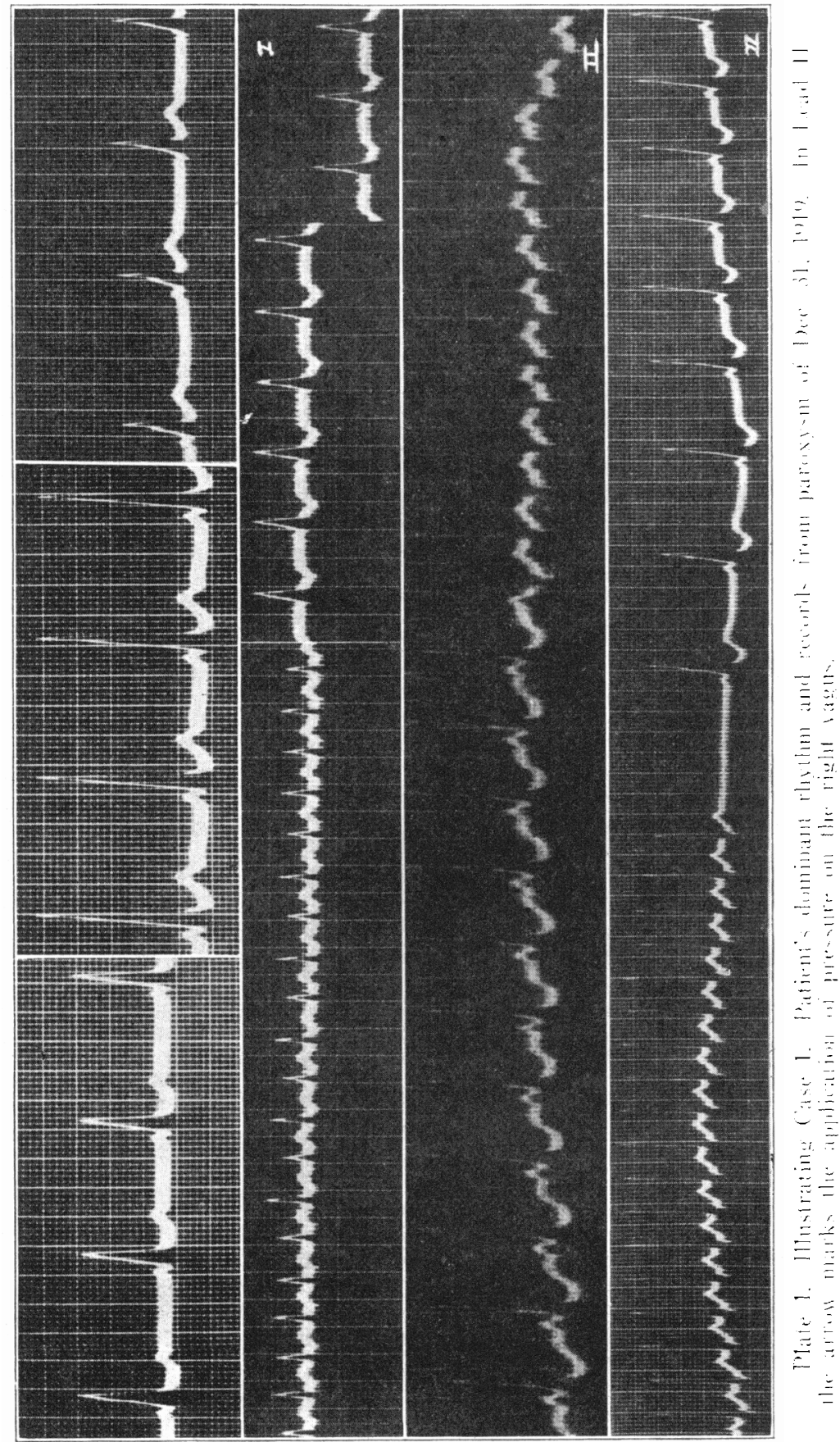

Downloaded From: http://archinte.jamanetwork.com/ by a New York University User on 06/01/2015 
forcefully; breathing is labored; the skin becomes flushed; he feels very warm, and perspires profusely. Occasionally, there has been pain about the heart.

Examination.-April 4, 1920, the patient visited the laboratory. The pulse rate varied from 72 to 80 , with sinus arrhythmia; blood pressure, 104/58; skin dry; pupils moderately dilated. After running eight times up and down a flight of twenty-four steps he returned to the laboratory saying "I am winded and it has not started yet." As he sat down the paroxysm began suddenly. Then the following observations were made:

Pulse Rate and Blood Pressure of J. W. (Case 1) During a Paroxysa

\begin{tabular}{ccc}
\hline Time & Pulse Rate & Blood Pressure \\
$11: 45$ & 74 & $104-58$ (Before exercise) \\
$12: 07$ & 184 & $144-80$ (After exercise) \\
$12: 10$ & 180 & $130-74$ (Breath held) \\
$12: 12$ & 180 & $115-76$ \\
$12: 14$ & 116 & $105-75$ \\
$12: 19$ & 104 & $103-65$ \\
$12: 40$ & 106 &
\end{tabular}

The pupils did not show any appreciable change; with the onset of the paroxysm the face became markedly flushed and a drenching sweat supervened. This attack was typical; variation is solely in duration.

Family History.-The family history is interesting. The paternal grandfather died suddenly at 48 ; he is said to have had a "tobacco heart." Three of the four sisters of the father have suffered from toxic goiter. A sister died of tuberculosis.

Previous History.-The patient himself has had measles, mumps, chickenpox and whooping cough when a small boy; diphtheria at 9 years and pneumonia at 13. There is no history of rheumatism or tonsillitis. Growth has been normal and general health has been excellent. There are no abnormal symptoms and the young man leads the life of an active college student, though handicapped by the irregular occurrence of the paroxysms.

Physical Examination. - The essential points in the physical examination were: Development and nutrition, good. No gross abnormalities.

Skin: Dry. Normal distribution of hair. Dermographia, central red line with a broader zone of blanching on either side.

Eyes: Pupils variable, often quite widely dilated.

Mouth and Throat: Negative.

Neck: No enlargement of thyroid.

Lungs: Negative.

Heart: Localized apex impulse in fifth interspace. Precordial dulness not increased. Sounds are of good quality and unaccompanied by murmurs. A 2 accentuated. Sinus arrhythmia with rate varying between 60 and 90 . Blood pressure: systolic, 100-126; diastolic, 58-76.

Abdomen: Negative.

Reflexes. Normal.

Fluoroscopic Examination.-No enlargement of heart. Quick, forceful pulsation, with rather jerky retraction of apex and unusually forceful pulsation of the aorta. Lungs were clear.

Roentgenogram of skull showed no abnormality of sella turcica. Blood cytology: normal. Wassermann reaction, negative. Basal metabolism: normal.

Reaction to Drugs.-Atropin, $0.03 \mathrm{gm}$., rate increased from 60 to 100 . The arrhythmia disappeared. In the electrocardiogram the P-R interval remained unchanged; $\mathrm{T}$ waves became inverted, returning to upright form in one hour. Pilocarpin, $0.12 \mathrm{gm}$.; no general reaction. No variations in details not seen normally. Epinephrin, $1 \mathrm{mg}$. No effect that could be attributed to it. 


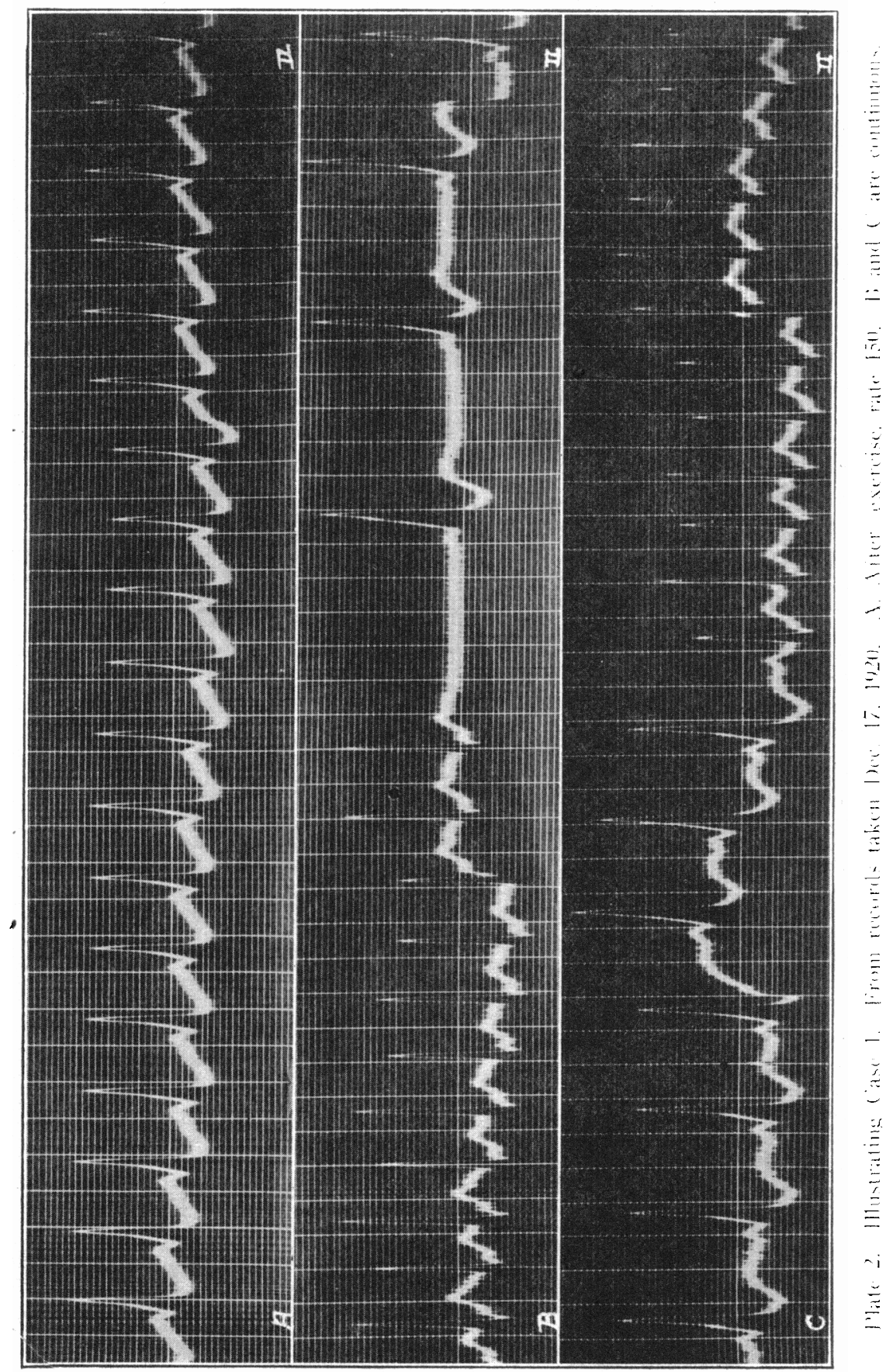

Downloaded From: http://archinte.jamanetwork.com/ by a New York University User on 06/01/2015 
Electrocardiograms. - The dominant rhythm has always been an A-V arrhythmia; $P-R$ interval, 0.08 second; width of $R, 0.12$ second; $T$ waves upright. Following exercise, atropin and the paroxysms, the $\mathrm{T}$ waves were inverted. The first beat of the paroxysm begins at the end of the normal interval of the fundamental rhythm, with an instantaneous doubling of the $\mathrm{P}-\mathrm{R}$ interval, halving of the duration of $\mathrm{R}$, and inversion or disappearance of $T$. The termination of the new rhythm is apparently by the production of a vagus block, prolongation of conduction time and failure of ventricular response. The rate of the paroxysm is from 180 to 195 ; variation in the $R-R$ intervals occurs and at times is due to variation, amounting almost to alternation, in the P-R interval. The figures in Plate 1 were taken in the latter part of December, 1919. Plate 2 was recorded Dec. 17, 1920.

CASE 2.-A woman, 52 years of age, had suffered for many years from hyperacidity and constipation. associated with alimentary stasis which is the result of marked visceroptosis. The examination of the cardiovascular system is negative, except for the disturbance of the cardiac mechanism. Ectopic beats are known to have been present for the past five years. The first attack of paroxysmal tachycardia occurred in February, 1919. Since then paroxysms have been of frequent occurrence; they often come on in the early morning hours or after meals and have varied in duration from a few minutes to eight hours. Vagus pressure has been tried by several physicians, none of whom have succeeded in stopping the paroxysm by that means. Relief has usually followed emptying the stomach by an emetic. The ectopic beats are seen to arise in the left ventricle. However, the electrocardiogram taken during the paroxysm indicates that the new rhythm arises in the A-V node and that reversal of the mechanism occurs; the type of curve suggests some defect in conductivity of the left branch of the bundle (Plate 3). The illustration was recorded during the afternoon of Oct. 27, 1919. The paroxysm began about $1 \mathrm{p} . \mathrm{m}$. Vagus pressure, aromatic spirits of ammonia, atropin $0.025 \mathrm{gm}$., and. finally, the passage of a stomach tube failed to arrest the paroxysm, which continued with occasional interruptions by a few normal beats until about 8 p. m. when it suddenly stopped. There was no cyanosis or dyspnea during the attack. The atropin caused marked dryness of the mouth and blurring of vision. Of late there have been no attacks. This is attributed to daily gastric lavage.

CASE 3.-This patient, a single man, aged 24 years, was referred April 6, 1920. with the complaint that on going to bed at night his heart became irregular and rapid, there being a succession of attacks during which the heart suddenly "started up," beat rapidly for a few minutes and then stopped abruptly. There was no pain, no shortness of breath and no symptoms other than the consciousness of the irregularity and this prevented sleep. The condition had been present since his discharge from the army in May, 1919, and was attributed to confinement in an office. He had influenza in January, 1919, while in France.

There has been no loss of weight or strength nor unusual fatigue. $\mathrm{He}$ plays volley hall and bowls regularly, without any symptoms referable to the cardiovascular or respiratory system. The hands and feet were often unduly moist, though the skin activity, in general, was not excessive; lacrimation or salivation has not occurred.

The family and the patient's past history are without significance. He has not had either rheumatic fever or tonsillitis. Venereal diseases were denied; the blood Wassermann reaction was negative.

Physical Examination.-Development and nutrition, good.

Skin: Generally dry, except the palms which were cold and clammy. Red line dermographia.

Eyes: Palpebral fissures abnormally wide; pupils dilated. Muscular reactions normal.

Mouth: Teeth good; no apparent pathology in tonsils. 
Neck: No enlargement of thyroid.

Lungs: Negative.

Heart: Apex beat in fifth interspace. No enlargement of percussion dulness. Sounds of good quality, without murmurs or abnormal accentuations. Rhythm, irregular due to the occurrence of frequent ectopic beats and short paroxysms of tachycardia. The systolic blood pressure varied between 90 and 112 and the diastolic from 60 to 75 ; during the paroxysms the systolic pressure fell to 80 ; the diastolic could not be read.

Abdomen: Negative.

Reflexes: Those at elbows and the knee jerks were exaggerated; plantar response, normal.

Blood: Cytology, normal except for a slight leukocytosis, 9,400 present during the second examination which was probably due to recent pharyngitis.

Paroxysms of tachycardia were of frequent occurrence. The taking of the pulse by the nurse or any of the laboratory procedures were sufficient to cause their appearance. They were usually of very short duration, lasting from a few seconds to one half hour, and averaged about 100 cycles. The average rate during the spontaneous paroxysm was 162 , with variation of about 0.02 second in the $R-R$ interval. The normal electrocardiogram is that characteristic of excessive sympathetic tone, with relatively large $\mathrm{P}$ and $\mathrm{T}$ waves and low R. Periods of slow nodal rhythm were seen occasionally. The paroxysms are believed to arise in the A-V node. In Lead I is seen a nodal beat, followed by a beat with a short P-R interval, then $\mathrm{P}$ is definitely behind $R$ when the paroxysm begins. At the end of the paroxysm was either a negative $P$ wave or the last beat showed a small upright $T$ and resembled many of the isolated nodal beats (Plate 4 ).

Epinephrin was without any effect, and while pilocarpin produced intense sweating there did not seem to be any specific effect on the electrocardiogram. However, following the injection of $0.03 \mathrm{gm}$. atropin a paroxysm came on in a few minutes which continued at a rate of 184 during one and one-half hours that the patient remained in the laboratory, and according to him did not stop for four hours.

CASE 4.-A farmer, aged 63, came to the hospital for prostatectomy. When first seen there was an irregularity due to occasional ectopic beats which arose at a low level in the atrium. A similar irregularity of the pulse was noted eight years ago when he had an eye infection. There has been no dyspnea on exertion, no thoracic pain, no edema of the extremities.

Physical Examination.-The prominent points of the physical examination were: The face was flushed and there was slight cyanosis of the lips. The lungs showed marked senile emphysema. The heart was not enlarged: there were systolic murmurs at both the apex and over the aortic area: the aortic second sound was accentuated. Blood pressure, 130/80. The first paroxysm was recorded Dec. 3, 1919. When questioned the patient said he had noticed slight discomfort about the heart but thought nothing of it. Subsequently many attacks were observed; it was not unusual for one to begin during a bedside examination. One attack is known to have lasted two hours. The patient was never conscious of the abnormal rhythm and the surgical history was uneventful.

From a comparison of electrocardiograms showing the isolated premature beats and those of the paroxysms, it seemed that the two arose at the same focus. The paroxysm is typical of the atrial tachycardias which arise well below the pacemaker. The illustration was obtained Jan. 24, 1920 (Plate 5, A). Pressure on the right vagus was exerted fourteen cycles before the effect noted. Although the paroxysm soon returned, this is doubtless an example of an ectopic rhythm which was influenced by vagus pressure.

CASE 5.-Man, aged 77 years, whose sole complaint was that he had had a bad taste in his mouth for the past five years. He has been short of breath on exertion for many years, but has never had any edema nor has he been conscious of any abnormal cardiac activity. 


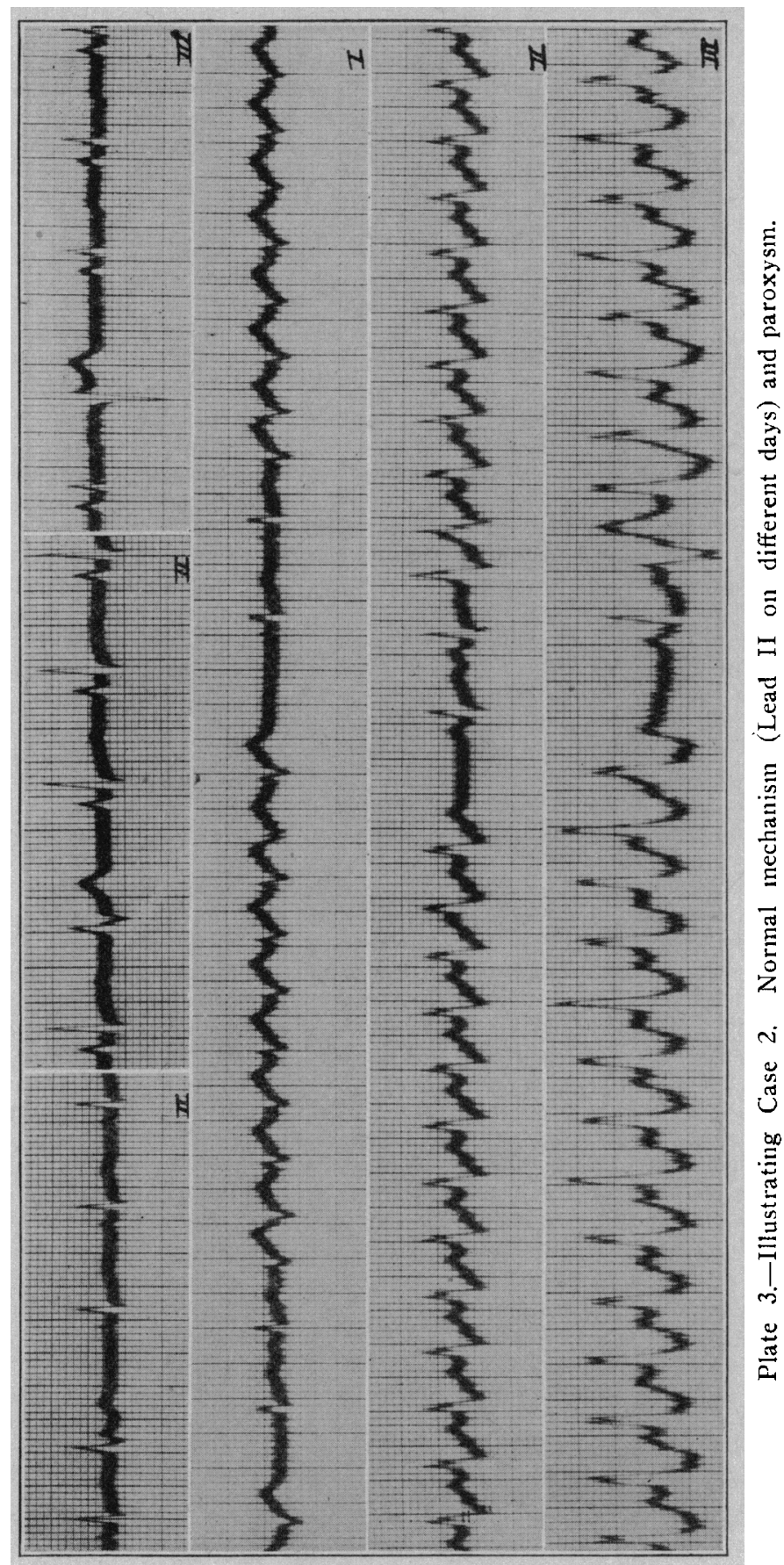

Downloaded From: http://archinte.jamanetwork.com/ by a New York University User on 06/01/2015 
Physical Examination.-Skin: Deep cyanosis of lips, tongue and oral mucosa.

Lungs: Senile emphysema.

Heart: Slightly enlarged to the left. Systolic murmur heard over entire precordium and transmitted to vessels of the neck. Rhythm, very irregular. Blood pressure, 200/80. Marked thickening of the peripheral vessels.

Abdomen: Liver enlarged and tender to pressure.

Extremities: Negative.

Fluoroscopic Examination.-Slight enlargement of heart. Localized enlargement of the arch of the aorta, an exaggeration of the usual senile knuckling. Marked opacity of the aorta.

Electrocardiogram.-Shows numerous ectopic beats which arise at two different levels in the atrium and in the left ventricle; also short paroxysms of tachycardia originating at a low level in the atrium. The last two cycles of the paroxysm suggest a shifting of the pacemaker to a higher level in the atrium (Plate 5, B). Because of the short duration of the paroxysms it was not possible to judge the effect of vagus pressure.

CASE 6.-The patient, a woman, aged 58 years, was in the hospital during the week of Jan. 3, 1921, suffering from pain in the lower right quadrant of the abdomen. A mass is said to have been palpated and an exploratory laparatomy advised, but the patient refused operation and left the hospital on the afternoon of January 10 . The next morning, about 4 o'clock, she was heard moaning by her niece with whom she was staying. She seemed very weak, was unable to speak for several hours, the pulse was almost imperceptible and the family believed she had suffered a stroke. A physician was called later. He noted the rapid heart action. The following day, January 12 , the patient was brought back to the hospital.

Physical Examination.-Examination was made at 7 p. m., the principal details of which were: A well developed and well nourished woman, whose appearance when first seen suggested that she was ten years older. She was able to recline without pillows, and was free from pain or any discomfort, except the consciousness of a rapid and pounding heart. The lips and fingers were slightly cyanotic. The lungs were negative; no moisture was present. In the abdomen, the liver edge was palpable, and there was tenderness on pressure over the cecum. The heart was enlarged a little to the left. Both sounds were very weak, unaccompanied by murmurs and without accentuations. The apex rate was 180 , and the rhythm was regular, and although the radial pulse was very weak, all beats came through to the wrist. Blood pressure. $95 / 82$. The electrocardiogram showed a regular tachycardia with a rate of 180. The type of curve was supraventricular and from careful examination of a considerable length of record it is believed to have had its origin in the lower part of the A-V node, and that reversal of the mechanism occurred. the $\mathrm{P}$ wave appearing as a small notch at the end of $\mathrm{R}$ (Plate 5. C). Holding the breath, pressure on the vagus in the neck and on both eyeballs was without any influence whatsoever on the rhythm.

Clinical Course.-Morphin was given and the patient rested well throughout the night. At noon, January 13 , the apex rate was still 180 ; blood pressure, $95 / 75$. Crepitant räles were heard at both bases, and the liver seemed larger than on the first examination. There was no dyspnea and she was comfortable except for the palpitation. Tincture of digitalis in 5 c.c. doses was given at 2 and at $4 \mathrm{p}$. m. Promptly, after the second dose, according to the patient, she vomited a large amount of "bile," the heart suddenly became slower and she dropped off to sleep. When seen at 7 p. m. the improvement in general appearance was quite srtiking. The pulse rate was 84 and the blood pressure 122/85. The electrocardiogram showed a normal mechanism.

January 15 a short paroxysm was reported but not studied. Examination the following day showed the pulse rate to be 80 and the blood pressure $145 / 75$; the lungs were free from moisture and the liver was not palpable. 


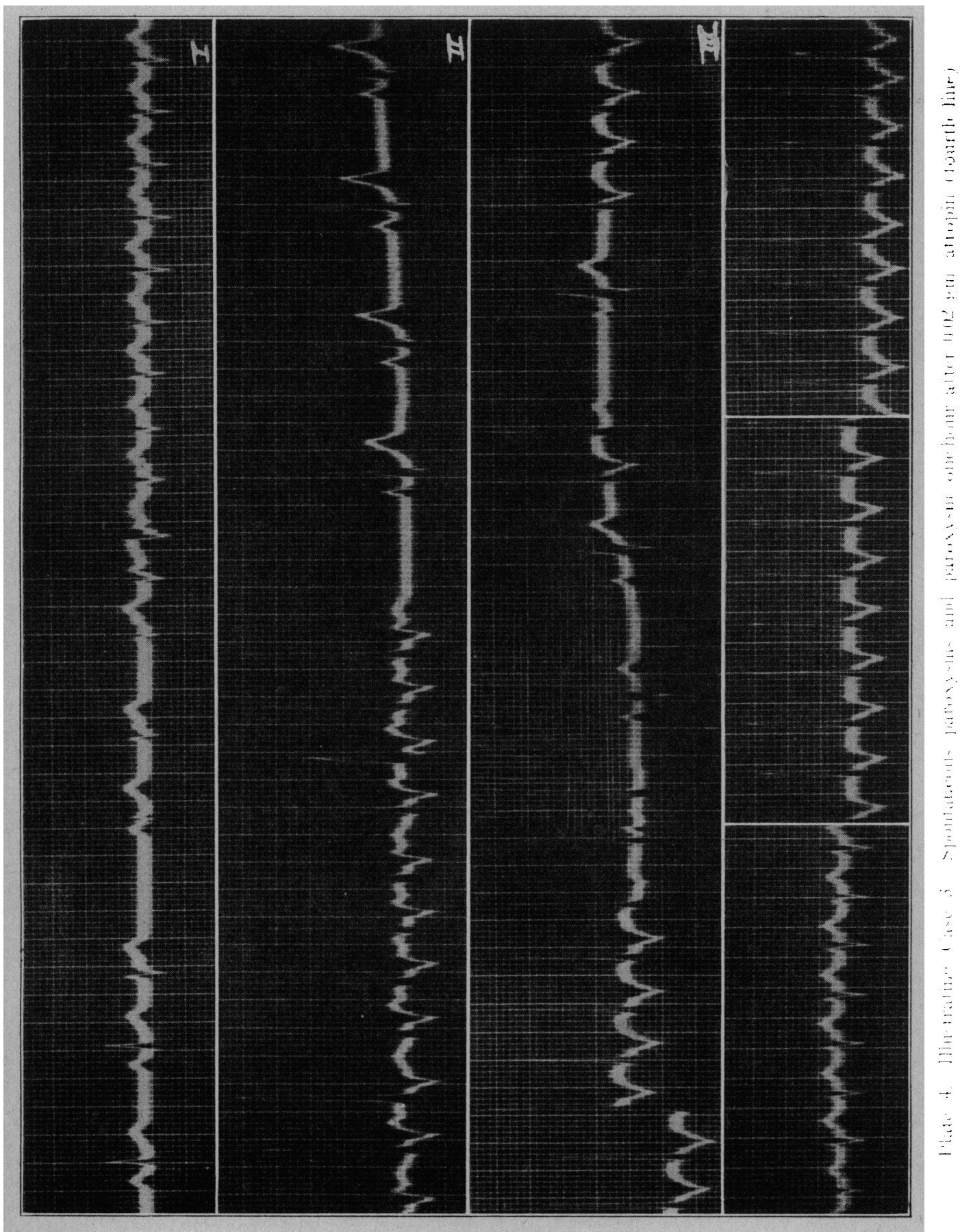

Downloaded From: http://archinte.jamanetwork.com/ by a New York University User on 06/01/2015 
Subsequent questioning brought information to the effect that since she was 15 years old the patient had suffered from such attacks which were characterized by very rapid beating of the heart. They frequently came on when at school; at times they were related to fatigue and again occurred during the night. No definite precipitating causes have been known. Fainting often occurred at the onset. Almost invariably the paroxysm has stopped after vomiting. Frequent examinations by numerous physicians have failed to reveal any signs of heart disease and physical and radiographic examinations during the week following the long paroxysm showed no evidence of disease either of the valves or of the myocardium. The previous paroxysm occurred last October. The one described was of the longest duration, having lasted for at least sixty hours.

This case is especially interesting in comparison with the first case cited, and recalls the analogy of Bouveret between epilepsy and paroxysmal tachycardia in that the individual is always under the menace of new paroxysms. The increasing duration of the attacks with the appearance of signs of myocardial failure during the latest one is of prognostic significance.

\section{NOMOTOPIC TACHYCARDIA}

Lewis ${ }^{2}$ has defined paroxysmal tachycardia as an affection in which the heart rhythm (sino-atrial nodal rhythm) becomes submerged and the heart responds to impulses formed at a more rapid rate in some other portion of its walls. Such a definition precludes the conception of a nomotopic tachycardia and the later statement that paroxysms arising in the sino-atrial node have not as yet been identified follows as a corollary. Likewise, Mackenzie ${ }^{3}$ states that paroxysmal tachycardia is a term to indicate the starting of the heart's contraction at an abnormal focus with a sudden increase in the rate of the heart. Donzelot, ${ }^{4}$ in a study of the pathology of paroxysmal tachycardia, has included all exaggerated accelerations of the heart which begin and end abruptly. His classification embraces: (a) Regular paroxysmal tachycardia, which results from displacement of the stimulus, though he says it is possible to conceive the existence of a paroxysmal tachycardia, "normotope," but "heterotype." (b) Auricular tachysystole, atrial flutter. (c) Irregular paroxysmal tachycardia, atrial fibrillation. Wenckebach has described nomotopic tachycardia and recently G. Galli ${ }^{5}$ reported a case with polygraphic records which he believed arose in the normal pacemaker, and has argued for nomotopic paroxsyms of neurogenic origin. Certain of his theses are worthy of criticism: I and IV. If vagus pressure is sufficient and prolonged it is usually successful in stopping the paroxysm: the Valsalva experiment reduces the

2. Lewis, T.: The Mechanism and Graphic Registration of the Heart Beat, New York, Paul B. Hoeber, 1920.

3. Mackenzie, J.: Principles of Diagnosis and Treatment in Heart Affections, London, Oxford Press, 1916.

4. Donzelot, E.: Les Tachycardies Paroxystiques: Etude Pathogénique, Ann. de méd. $11: 155,1914$.

5. Galli. G.: Sur les Mécanisme de Terminaison et de Début des Accès dans 1a Tachycardie Paroxystique (Nomotope), Arch. d. mal. du coeur 12:289, 1919. 


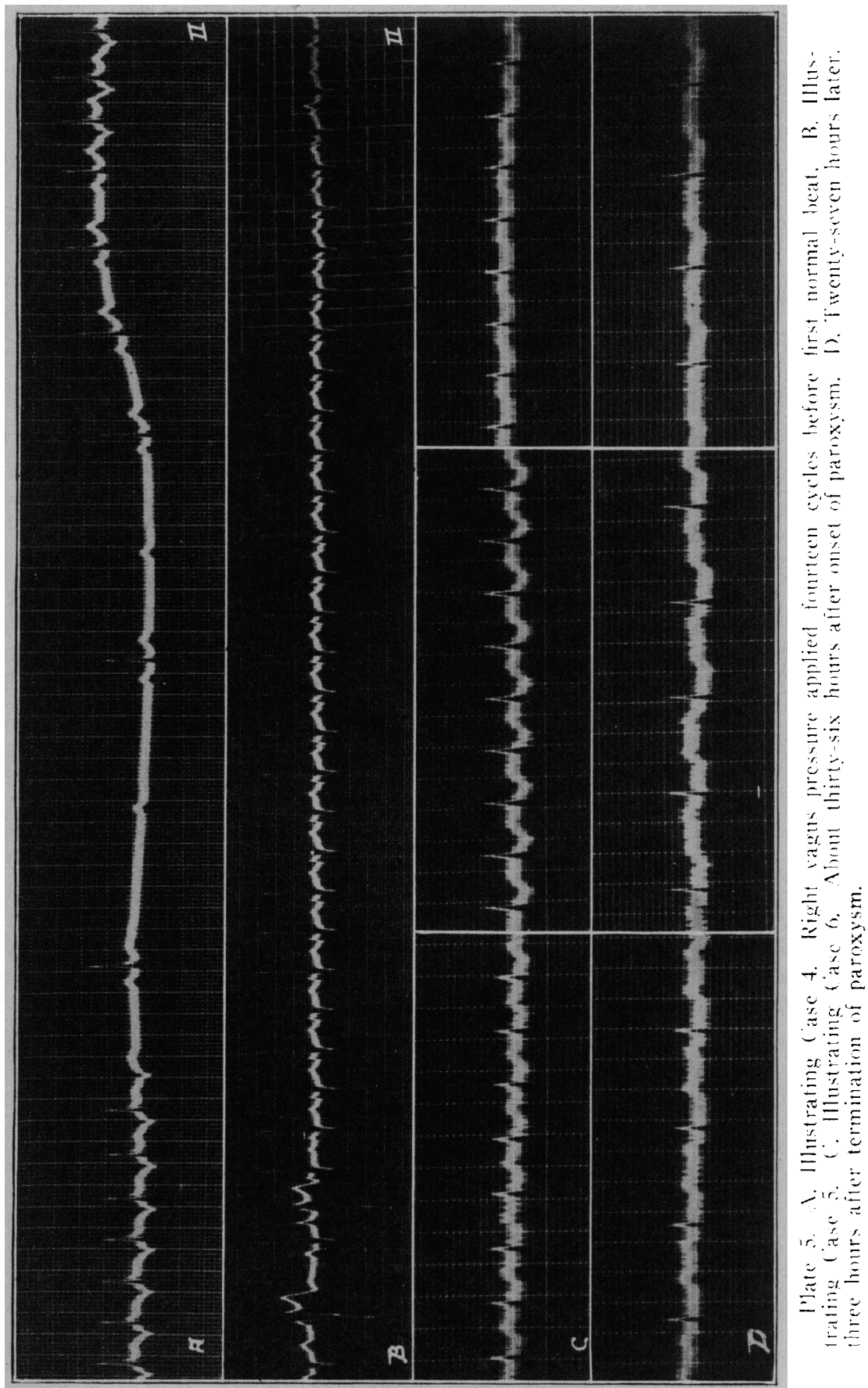


frequency of simple tachycardia and often arrests a paroxysm: those tachycardias not thus relieved are of heterotopic origin. The failure of vagus pressure on the basis of inadequate stimulation is difficult to answer. Surely but few continue vagus pressure for thirty minutes. The common practice is to apply pressure until the heart action is altered or the discomfort produced demands its discontinuance. It is the usual experience of those who resort to this procedure that in some cases it is apparently successful while in others there is no effect. In Case 1 of the present series the effect of vagus pressure was doubtful and in the case of Carter and Wedd ${ }^{6}$ no pressure that could be endured influenced the paroxysm. On the other hand, there are numerous records of unquestioned heterotopic tachycardias which are definitely affected by stimulation, for example, Case.4. In Case 3 the patient had long successfully practiced the Valsalva experiment, but the effect of vagus pressure is difficult to interpret because of the recurring short paroxysms, and the fact that there was no change in the electrocardiogram. III. Atropin in sufficient dosage may incluce a paroxysm of cachycardia. It did in Case 3 , but the paroxysm arose at the $A-V$ node, not the sinus node. $V$. An emotion may bring forth a paroxysm. But again, it may be a heterotopic rhythm, as in Case 3. VII. There occurs at times an abrupt passage from normal rhythms to that of simple tachycardia. This presupposes proof of the exact nature of both phases. From observation alone of the movements of the galvanometer string during the paroxysm in Case 1 that seemed to occur. but examination of the record showed that without any pause, the change in rate from 115 to 188 followed a shift of pacemaker to a higher level in the auricle, with prolongation of $\mathrm{A}-\mathrm{V}$ conduction and other changes in the electrocardiogram. X. Rhythmic oscillations may occur during a paroxysm; this is characteristic of sinus activity. The slow mechanism in Case 1 is an $\mathrm{A}-\mathrm{V}$ arrhythmia. It is not possible to deny that fluctuation might occur in nodal tachycardias, though this rarely, if ever, occurs in any marked degree. VIII. The electrocardiogram usually shows a negative $\mathrm{P}$ wave and shortened conduction time: but $P$ is not always inverted even in cases of evident nodal rhythm: moreover the eye accustomed to the perusal of electrocardiograms may be occasionally deceived, and finally the electrocardiogram is often difficult to interpret and ambiguous. All this is admitted, and likewise the conclusion that because the electrocardiogram shows most tachycardias to be heterogenetic, it is unjust to insist that only extrasinusal tachycardias may occur. However, when the electrocardiogram fails to

6. Carter, E. P., and Wedd, A.: M.: Report of a Case of Paroxysmal Tachycardia Characterized by Unusual Control of the Fast Rhythm, Arch. Int. Med. 22:571 (Oct.) 1918. 
show the origin of the rhythm, to what shall one turn? Polygraphic records may be more simple of interpretation, but who shall vouch for their authority?

Shortly after the appearance of the paper by Galli, a case of paroxysmal tachycardia was reported by Boden " in which during the paroxysm, the $\mathrm{P}$ wave of the electrocardiogram was similar to that in the normal, but with an increase in negativity in the ventricular complex. After atropin the electrocardiogram was exactly like that during the paroxysm, though the rate increased to only 144 while that of the paroxysm was 170 . This was interpreted as a case of nomotopic tachycardia resulting from dominance of the accelerator system. In the discussion of the case of paroxysmal tachycardia with voluntary control reported by Carter and Wedd, while the term nomotopic was not used, it was stated that there could be no ectopic focus wholly removed from neurogenic control which was responsible for the fast rhythm. More recently White ${ }^{8}$ has considered this subject in a study of a case in which there were short "paroxysms," but no abrupt onset or offset in the rapid rhythm. The $\mathrm{P}$ wave remained upright and fell back on $T$ and there were slight variations in the intervals between beats at the height of the paroxysm, the maximum rate of which was about 188 . This was called "paroxysmal tachycardia, arising in or very near the sino-atrial node and not showing an absolutely abrupt onset or offset." From the definitions given and the work cited the existing confusion is apparent. In the light of Lewis' later work, closer relations between extra-systoles, paroxysmal tachycardia, flutter and fibrillation are indicated, and it may well be that Donzelot in his clinical classification five years ago wrote better than the experimental knowledge of the time warranted. But to introduce as a paroxysmal tachycardia an altered mechanism which is neither heterogenic, ectopic nor paroxysmal in the common clinical meaning of having an abrupt onset and offset, is to further complicate the situation by bringing into the category simple tachycardias.

As to the nature of paroxysms of tachycardia, the conclusion is inevitable (Lewis) that the paroxysm is composed of a series of extrasystoles. Extrasystoles definitely known to arise in either the S-A or A-V node are rare, though their possibility or existence is not denied. (The term "extrasystole" is here used following the terminology of Lewis. Theoretically, the only true extrasystole not an ectopic beat is an interpolated sinus extrasystole. Inasmuch as practically all so-called

7. Boden, E.: Uber den Einfluss der langen Hèrznerven auf die Form des Elektrocardiogramms in einen Fall von Paroxysmaler Tachycardie, Deutsch. Arch. f. klin. Med. 130:249, 1919.

8. White, P. D.: Clinical Observations on Unusual Mechanisms of the Auricular Pacemaker, Arch. Int. Med. 25:420 (May) 1920. 
extrasystoles are in reality ectopic beats, it would simplify a discussion of heterogenetic impulses and clarify their inter-relation if the former term could be dropped from the literature.) The occurrence of ectopic beats in the tachycardias under consideration may be mentioned at this point. In Case 1 no isolated abnormal beats have ever been seen. In Case 2 ectopic beats arising in the left ventricle have been frequently recorded; the paroxysm, however, was probably of supraventricular origin. Nodal beats were frequent in Case 3 and often occurred in pairs. In Case 4 ectopic beats arising at a low level in the atrium were constantly present, often giving rise to a coupled rhythm and from the conformation of the curves it appeared that the paroxysm arose at the same focus. In Case 5, although both ventricular and atrial ectopic beats occurred, the only paroxysms recorded were of the atrial type. No ectopic beats have been noted in Case 6 , but the patient was under observation only ten days. Paroxysms which arise in the $\mathrm{A}-\mathrm{V}$ node are known. Case 3 affords another example. There is no difficulty in this case, likewise in Cases 4 and 5 , in seeing the paroxysm as a succession of extrasystoles. If extrasystoles can occur in the sinus node what is there to prevent a series of them? And if increased irritability may occur abruptly in the $\mathrm{A}-\mathrm{V}$ node resulting in a succession of beats that constitutes a paroxysm, what prevents such an accident at the sinus node? Doubtless the more complete, at least better known, regulatory mechanism at the sinus node is an important factor.

In any criteria for paroxysmal tachycardia it would seem well to insist that the essential characteristic of the new rhythm shall be its abrupt onset and termination. A recent observation is that of a woman of 50 years of age who had had a finger nail removed, with cocain for the local anesthetic. Following the operation the pulse rate increased to 160 and she thought that the rapid and forceful heart action came on suddenly as she was being helped into bed. The cardiac mechanism was watched with the galvanometer for two hours. The electrocardiogram at this time and later showed that the rapid rhythm arose at the sinus node. The systolic pressure varied between 200 and 185 and the diastolic remained at about 110 . From subsequent observations it appeared that the normal pressure was $150 / 85$. The rate remained quite constant for about three hours; vagus pressure produced temporary slowing but did not break the rhythm. It seemed for a time as though this might be a sino-atrial paroxysmal tachycardia, but after four hours the rate was 140 and after six hours 120 ; the next morning it was 100 , and during the two following days varied between 80 and 124. In experimental accelerator stimulation sino-atrial rates from 150 to 250, and occasionally higher, have been obtained. In Case 1 the nodal rhythm following exercise reached the rate of 150 . The par- 
oxysms reported showed rates varying between 120 and 195 . It is not necessary to consider any rhythm which shows neither displacement of the stimulus nor an abrupt beginning or ending as other than a simple tachycardia. Rate alone can have no place as a criterion for paroxysmal tachycardia.

The difficulties of establishing a nomotopic paroxysm by means of the electrocardiogram are manifold. It is necessary to consider, and to differentiate, if possible, not only the intrinsic changes that may accompany the new rhythm but changes that may be due to increase in rate, stimulation of the cardiac nerves or changes in chemical environment, associated with or dependent on either of the foregoing. ${ }^{9}$ No analysis of the paroxysm is reliable unless the onset and termination have been recorded. The record of Boden is wanting in this respect. That of Galli need not be considered because of the inadequacy of polygraphic records. In Case 2 , if one were to consider only the fully developed new rhythm, especially Lead II, it would not be difficult to establish a nomotopic tachycardia; the wave preceeding $R$ could be regarded as $P$ and the conduction time would be normal; the small thickening after $\mathrm{R}$ would be called $\mathrm{T}$ and the diminished size, likewise the notching and the widening of $R$ would be attributed to alteration in nerve control or chemical environment. But the facts that after a brief respite the paroxysm invariably begins without an atrial wave, and that the definite wave between the spikes spaces accurately as a $T$ wave, cannot be dismissed. Another possible interpretation is that the paroxysm arises in the upper part of the right branch of the bundle and independent atrial contractions occur at the same rate. But from consideration of all the facts, a nomotopic tachycardia is impossible. In Case 1 there is an abrupt onset of a rapid rhythm apparently with displacement of the pacemaker to a higher level, lengthening of $\mathrm{A}-\mathrm{V}$ conduction from 0.08 to 0.16 second, a lower and narrower $R$ wave and absence of $T$. As has been noted, the paroxysm of tachycardia is but one feature of a temporary but profound sympathetic stimulation. This question arises, are these changes in the electrocardiogram specific for the new rhythm, or are they the result of sympathetic stimulation acting on the original rhythm, an exaggeration of those seen after the earlier exercise. The inversion and disappearance of $\mathrm{T}$ has been seen before; the lowering of $R$ and shortening of ventricular response may be due to the increase in rate. Sympathetic stimulation tends to decrease A-V conduction and increase in rate to prolong it. The fact that the conduction time in this first cycle is practically double that of the preceding is significant and the later lengthening may be associated with increased rate. If as

9. Mines, G. R.: On Dynamic Fquilibrium in the Heart. J. Physiol. 46: 349. 1913. 
Lewis says the changes seen during the paroxysm are an intrinsic part of the new mechanism then this is a new rhythm arising at a higher level in the atrium and details suggest that its origin may be the $\mathrm{S}-\mathrm{A}$ node. In the absence of authoritative criteria nomotopic paroxysmal tachycardia cannot be proved in clinical electrocardiograms, but to deny the possibility of the same is to deny to the sinus node properties of irritability and rhythmicity present in the $\mathrm{A}-\mathrm{V}$ node, also a homogenetic centre, and other loci in the heart where these functions are normaliy present in less rlegree, and surely this requires proof.

\section{THE RÔLE OF THE EXTRINSIC NERVES}

The relation of the extrinsic nerves to cardiac irregularities has been extensively investigated. The attempt to control paroxysms of tachycardia by vagus pressure has long been practiced. The actual effects are debatable. While there are those who believe the procedure infallible, according to Lewis either there is no effect or the new rhythm abruptly ends and the normal rhythm is resumed. In Case 1, in which vagus control might be expected, on one occasion the paroxysm stopped twenty cycles after the application of pressure; prolongation of conduction and failure of ventricular response occurred, a common result of vagus stimulation. But at another time the paroxysm terminated in an exactly similar way when no pressure was used and the patient made no effort to stop the paroxysm. The same mechanism was seen for the third time, when the individual held his breath to stop the attack. In the case of Carter and Wedd, likewise a possible nomotopic tachycardia, there was lengthening of the P-R interval of the last cycle and failure of ventricular response terminated the paroxysm, although this could not be brought about by vagus pressure. It would seem in these two cases, both of which were due to predominant accelerator action, as though the normal rhythm was restored by a resumption of vagal tone, but that this did not occur as the result of interference from without. In Case 3, in which the paroxysms were definitely of sympathetic origin, one paroxysm continued for fifty-nine beats, being of longer duration than many, and ended abruptly without any change in the electrocardiogram. In Case 4, an atrial tachycardia, vagus pressure did apparently break the ectopic rhythm by the production of block.

The accelerator system has likewise received much attention. Bouveret ${ }^{10}$ in 1889 , from two cases of his own and from similar ones in the literature, described the malady that has since borne his name. essential paroxysmal tachycardia, which occurred in individuals said to be free from any disease of the heart, thyroid or central nervous system.

10. Bouveret, L.: De la Tachycardie Essentielle Paroxystique, Rev. de méd. 9:753, 1889 . 
Because of normal findings in the intervals between attacks he argued that the trouble must come from a profound disturbance in the innervation of the heart and believed that the mechanism was by excitation of the accelerator nerves, probably in the intracardiac ganglia. The etiology included a combination of physical and cerebral causes; physical fatigue, emotion and sense of cold were given as precipitation causes of the paroxysms. Rothberger and Winterberg, ${ }^{11}$ studying the effect of stimulation of the accelerator nerves and sympathetic ganglia, found the usual reaction to be a simple tachycardia with characteristic changes in the electrocardiogram; in one instance after stimulation of the left accelerator a negative atrial wave appeared without increase in frequency; following simultaneous stimulation of both accelerators $T$ almost entirely disappeared and when present was a negative wave. In a later work they produced a heterotopic tachycardia by sympathetic stimulation in a heart previously treated with barium chlorid. Donzelot reported extrasinusal or heterotopic tachycardias from stimulation of the left stellate ganglion. Levy ${ }^{12}$ has shown the occurrence of ectopic ventricular beats after accelerator stimulation in cats which were under light chloroform anesthesia. Clerc and Pezzi ${ }^{13}$ obtained heterotopic tachycardias arising in various levels of the $A-V$ node after excitation of the accelerator nerves, but instead of faradic stimulation, they resorted to strontium chlorid and nicotin for exciting agents. The conclusion of Lewis from his consideration of experimental and clinical cases is that it has not been shown that abnormal nerve impulses playing upon a normally nourished heart may be responsible for extrasystoles or higher types of disorders, but that nervous impulses playing upon an irritable heart may provoke such reactions.

Galli ${ }^{14}$ recently reported a case in which paroxysms are said to have come on after atropin, and which he believed to be conclusive evidence of vagus control of the paroxysm. Lewis emphasizes the fact that this occurred in a patient subject to paroxysms. Moreover, in this case the small dose of atropin generally given and the delayed response cast some doubt on the atropin effect. One $\mathrm{mg}$. hypodermically is a small dose, and the tachycardia did not develop for forty-three minutes. The atropin effect ordinarily has begun to wear away by this time. The only convincing record is that in his Figure 5, in which the paroxysm came on fifty minutes after the administration of 30 drops by mouth

11. Rothberger and Winterberg: Ueber die Beziehungen der Herznerven zur Form des Elektrocardiogramms, Arch. f. d. ges. Physiol. 135:506, 1910.

12. Levy, A. G.: The Exciting Causes of Ventricular Fibrillation in Animals under Chloroform Anesthesia, Heart 4:319, 1912.

13. Clerc, A., and Pezzi, C.: Le Rythme Septal du Coer, Arch. d. mal. du coeur 13:103, 1920.

14. Galli, G.: Attacks of Paroxysmal Tachycardia Following Atropin, Heart, $7: 111,1920$. 
and persisted for two and one-quarter hours, and here it is the comparative duration that is important. In Case 3 of this series shortly after the administration of $2 \mathrm{mg}$. atropin a paroxysm occurred at a higher rate than the spontaneous paroxysms, and persisted without interruption for four hours, during which time no usually successful maneuvers would stop it. The higher rate in this paroxysm may have been due to the fact that although the spontaneous paroxysms resulted from heightened accelerator action the vagus still exerted some influence, which was removed by atropin. That a slight mental disturbance occurred may have indicated unusual susceptibility to this drug, but the cardiac response was such as to leave no doubt that the abolition of vagus tone was a definite factor in the long paroxysm.

The position of Lewis, which postulates an underlying toxemia rendering the heart abnormally susceptible to unusual activity of the extrinsic nerves, is incontrovertible, for who can prove that there is no subtle poison at work. It remains to inquire into the possible nature of the toxins. Danielopolu, ${ }^{15}$ accepting the doctrine that paroxysmal tachycardia cannot originate in an intact myocardium, described an interesting case of exophthalmic goiter in which there were paroxysms of tachycardia associated with unilateral exophthalmos. According to his explanation, simultaneous stimulation of both accelerators occurred. that of the right gave rise to the exophthalmos and the left, to a heterotopic tachycardia. For the latter he assumed a lesion in the bundle of His which was in a hyperexcitable state and the toxic product of the thyroid gland acted as the exciting agent. A few cases of paroxysmal tachycardia have been reported in which histologic findings were negative, and in the case of thyroid disease it does not appear necessary to presuppose an organic lesion; moreover, this is unlikely in the early stages of the disease. Besides the affections attributed more or less definitely to the thyroid there remains the entire gamut of disorders of the autonomic nervous system. Cases 1 and 3 of this series hardly fit into any of the groups included under exophthalmic goiter or in that of the irritable heart, nor is there sufficient reason to believe that early organic heart disease exists. In the first case the paroxysm is only an incident in the violent reaction to sudden sympathetic stimulation; the drenching sweats are the outstanding feature for the patient. In the other case, the dilated pupils, sweating palms, dermographia and unstable cardiac mechanism constitute a familiar clinical picture, of which the paroxysms so easily provoked are only a part. But these individuals belong somewhere among the confused syndromes called

15. Danielopolu, D.: Accés de Tachycardie Paroxystique avec Exophthalmie Unilaterale chez une Ancienne Basedowienne, Compt. rend. Soc. de biol. 79: $103,1916$. 
endocrinopathies. It is hardly advisable to emphasize Case 6 in this connection, except to recall that there is a definite history of paroxysms of tachycardia extending over a period of forty-three years and that even today there is no demonstrable evidence of heart disease in the usual meaning of that term, and to suggest that this patient belongs to the same group. In such conditions is not the consideration of toxic substances rendering centers abnormally susceptible to nervous influence which precipitate the disturbances merely calling one thing by two names, or perhaps attempting to push the question further back into the chemical pathology of the autonomic system? And may it not be that when this pathology shall be understood, there will stand one group of paroxysmal tachycardias due to faulty nervous control as Bouveret has written, the fault being whatever may come to be recognized as pathologic in the autonomic nervous system?

\section{SUM MARY}

Six cases of paroxysmal tachycardia are reported. Two are in elderly individuals in whom there are signs of degenerative changes involving the heart and aorta. This is a frequent association and the paroxysms are believed to be expressions of high irritability in inflammatory foci in the muscle wall. A third case is an example of a group of paroxysmal tachycardias that are usually considered to be of reflex origin. The visceroptosis and alimentary stasis are of long standing. The recent appearance of paroxysms where formerly ectopic beats alone occurred indicates an altered response on the part of the heart. The direct relation between the paroxysms and the alimentary condition is apparent and suggests that the paroxysms are analagous to those produced by ligation of the coronary arteries; because of mechanical displacement embarrassment of the coronary circulation results and there may have occurred changes in the heart muscle, not detectable by physical signs, which render the myocardium less tolerant of interference with its blood supply. Three of the cases seem to fulfill the requirements of the "maladie de Bouveret." There is no evidence of organic heart disease, nor of Graves' disease or so-called hyperthyroidism. The two young men are suffering from some disorder of the autonomic nervous system in which transient predominance of the accelerator division frequently occurs. In this connection the role of the extrinsic nerves of the heart in paroxysms of tachycardia has been considered, and it is believed unnecessary to postulate the existence of an underlying toxemia which renders the heart abnormally susceptible to nerve impulses, for both of these factors may well be one and the same thing, and it is hardly necessary at present to attempt to differentiate neuro- from chemical pathology in the autonomic nervous system. 
Concerning nomotopic paroxysmal tachycardia there is much confusion in the literature and the most authoritative definitions of paroxysmal tachycardia do not include even the possibility of its existence. It is suggested that the first requirement of any paroxysm shall be an abrupt onset and termination. The necessity of recording the onset of a paroxysm for its correct analysis has been pointed out. In the first case reported, in which the dominant rhythm has its origin in the $A-V$ node, with the onset of the paroxysm there was a sudden shifting of the pacemaker to a higher level in the atrium and from the character of the electrocardiogram the new rhythm might have originated in the S-A node. However, nomotopic tachycardia cannot be established by clini- cal electrocardiograms. Paroxysms of tachycardia are now regarded as successions of extrasystoles. The existence of sinus extrasystoles is recognized. But even in the absence of demonstrable relationship between such beats and the paroxysm in any given case the negation of the possibility of a nomotopic paroxysmal tachycardia does not seem justifiable. 